\title{
Administração de toxina botulínica A e a orquiectomia no tratamento da hiperplasia prostática benigna do cão
}

\author{
Botulinum toxin A administration and orchiectomy in the \\ treatment of canine benign prostatic hyperplasia
}

\author{
Giuliano Queiroz Mostachio ${ }^{\mathrm{I}}$ Maricy Apparício ${ }^{\mathrm{II}}$ Tathiana Ferguson Motheo ${ }^{\mathrm{II}}$ \\ Aracélle Elisane Alves ${ }^{\text {III }}$ Wilter Ricardo Russiano Vicente ${ }^{\text {II }}$
}

\section{RESUMO}

A fisiopatologia da hiperplasia prostática benigna (HPB) não está totalmente compreendida, no entanto, a diidrotestosterona é o principal hormônio envolvido. Recentemente, o efeito da toxina botulínica $A$ (TB-A) foi investigado, mostrando que esta induz atrofia do parênquima e redução do volume prostático. Com base nisso, este estudo teve como objetivos comparar os efeitos da administração da TB-A com a orquiectomia no tratamento da HPB, além de avaliar os efeitos da TB-A sobre a libido e qualidade do sêmen. Para tanto, 16 cães adultos foram submetidos à castração ou administração de $500 U$ de TB-A, e avaliados durante 16 semanas. A orquiectomia mostrou-se um excelente tratamento, promovendo redução de $80 \%$ do volume prostático. Aplicação da TB-A não ocasionou alterações na libido, ereção ou qualidade e características seminais. Por outro lado, reduziu significamente o volume da próstata. Os resultados sugerem que a administração intraprostática de TB-A é um tratamento alternativo efetivo e sem efeitos colaterais para cães destinados a programas de reprodução. No entanto, esta terapia apresenta reduções inferiores do volume prostático, quando comparada com a orquiectomia.

Palavras-chave: cão, próstata, HPB, neurotoxina, sêmen, orquiectomia.

\section{ABSTRACT}

The pathogenesis of benign prostatic hyperplasia (BPH) is not completely known, however, dihydrotestosterone is a hormone involved in enlargement of the prostate. Recently, the effect of botulinum toxin A (BT-A) was investigated and has shown to induce atrophy of the gland. Based on that, the aims of this study were to compare the effects of administration of BT-A with orchiectomy in the treatment of $B P H$, in addition to assessing the effects of BT-A on libido and semen quality. Therefore, 16 sexually intact male dogs were submitted to orchiectomy or administration of $500 U$ BT-A, and evaluated for 16 weeks. Orchiectomy presented excellent results, reducing the prostate volume up to $80 \%$. Administration of BT-A did not interfered on libido, erection or semen characteristics. On the other hand, reduced significantly the prostate volume. The results suggest that intraprostatic injection of $B T$-A is an effective alternative treatment without adverse effects for valuable breeding dogs with BPH. However, this therapy showed lower reduction of prostate volume when compared to orchiectomy.

Key words: $d o g$, prostate, BPH, neurotoxin, semen, orchiectomy.

\section{INTRODUÇÃO}

Diante do aumento da expectativa de vida dos cães, problemas relacionados à próstata, especialmente a hiperplasia prostática benigna (HPB), vêm adquirindo importância. A etiologia da doença no cão é incerta, no entanto, pesquisas demonstraram que a próstata está sobre influência da inervação autonômica e aos seus neurotransmissores, sendo que a acetilcolina promove a estimulação do crescimento da próstata, assim, por essas razões, estudos têm confirmado que a toxina botulínica do tipo A (TB-A) é efetiva para controlar os componentes mecânicos e dinâmicos da HPB (DOGGWEILER et al., 1998; KUO, 2005).

A orquiectomia é o tratamento de escolha para os cães acometidos pela enfermidade, no entanto, naqueles destinados à reprodução ou onde há contraindicação cirúrgica, a terapia conservativa é de grande importância. Em homens, a administração

\footnotetext{
'Departamento de Clínica Cirúrgica de Pequenos Animais, Centro Universitário de Rio Preto (UNIRP), BR 153, Km 69, 15093-450, São José do Rio Preto, SP, Brasil. E-mail: wise_uel@yahoo.com.br. Autor para correspondência.

${ }^{I}$ Departamento de Reprodução Animal, Faculdade de Ciências Agrárias e Veterinárias (FCAV), Universidade Estadual Paulista (UNESP), Jaboticabal, SP, Brasil.

IIIDepartamento de Cirurgia, Faculdade de Medicina Veterinária (FAMEV), Universidade Federal de Uberlândia (UFU), Uberlândia, MG, Brasil. Recebido 24.08.14 Aprovado 29.01.15 Devolvido pelo autor 18.05.15 CR-2014-1253.R1
} 
de TB-A, intraprostaticamente, tem propiciado excelentes resultados, com uma rápida e segura redução do volume do órgão, além de ser adequada naqueles com riscos por problemas pré-existentes (MARIA et al., 2003; KUO, 2005). Entretanto, a influência dessa neurotoxina foi pouco estudada no cão, existindo poucos relatos de sua utilização (CHUANG et al. 2006b; MOSTACHIO et al., 2012). Segundo CHUANG et al. (2006b), as avaliações histopatológicas do tecido evidenciaram aumento no número de células apoptóticas e subsequente redução do volume prostático. Vale também ressaltar que grande parte dos fármacos utilizados na terapia conservativa em cães, como os inibidores da enzima $5 \alpha$-redutase e agonistas/antagonistas de GnRH, provocam efeitos colaterais indesejáveis, entre eles: redução do volume seminal e aumento de anomalias morfológicas (BARSANTI \& FINCO, 1995). Portanto, em cães destinados à reprodução, a TB-A poderia ser uma alternativa.

Sendo assim, este estudo teve como objetivo comparar os efeitos da aplicação intraprostática de TB-A com a orquiectomia no tratamento da HPB canina. Além de investigar as implicações da administração da TB-A em relação à qualidade seminal, libido, concentrações séricas de diidrotestosterona (DHT) e testosterona, volume e diâmetro prostático no cão com hiperplasia prostática.

\section{MATERIAL E MÉTODOS}

Foram utilizados 16 machos não castrados da espécie canina, sem raça definida, pesando $21,9 \pm 11,1 \mathrm{~kg}$ (média \pm desvio padrão), com idade entre três e 11 anos $(7,0 \pm 2,29)$, os quais possuíam sintomatologia clínica compatível com HPB e que, à palpação retal, apresentavam aumento de volume prostático simétrico, indolor e com superfície lisa ao toque. Em todos os cães selecionados, o diâmetro prostático na projeção radiográfica lateral foi superior a $70 \%$ da distância entre o promontório sacral e o púbis, e/ou o volume prostático mensurado por ultrassom maior do que $15 \mathrm{~cm}^{3}$. Em adição, duas semanas antes da seleção dos animais que compuseram os grupos experimentais, realizouse a punção aspirativa com agulha fina guiada por ultrassom. A análise da citologia aspirativa do tecido prostático dos animais selecionados, evidenciou grande número de células semelhantes às normais, com citoplasma basofílico, ligeiramente granular, contendo algumas vacuolizações, e com aumento da relação núcleo: citoplasma, características sugestivas de HPB. A avaliação do fluído prostático revelou ainda ausência de bactérias e células inflamatórias.

Os cães foram divididos aleatoriamente em dois grupos, contendo oito animais cada. No grupo 1
(GI), os animais foram submetidos à orquiectomia. No grupo 2 (GII), o cães receberam 500 unidades (U) de TB-A, dividida em duas aplicações de igual volume, administradas em cada lobo prostático. Todos os animais foram submetidos previamente a jejum alimentar e hídrico de 12 e quatro horas, respectivamente. Os animais foram pré-medicados com levomepromazina (1mg $\mathrm{kg}^{-1}$ ) associado a tramadol $\left(2 \mathrm{mg} \mathrm{kg}^{-1}\right)$ pela via intramuscular. Transcorridos 15 minutos, a anestesia foi induzida com propofol $\left(5 \mathrm{mg} \mathrm{kg}^{-1}\right)$ pela via intravenosa e os animais mantidos em anestesia geral inalatória com isofluorano. Após anestesia, os animais do GI foram orquiectomizados de maneira convencional e os do GII receberam a administração de TB-A. Cada frasco de TB-A foi diluído com $4,0 \mathrm{~mL}$ de solução salina $0,9 \%$, obtendo uma concentração de $125 \mathrm{U}$ para cada $\mathrm{mL}$ de solução. Com o auxílio do aparelho de ultrassom, introduziu-se agulha de calibre $(60 \times 10$ ou $80 \times 10 \mathrm{~mm}$ ) adequado ao porte do animal no interior da próstata, e cada lobo dos animais do GII receberam $250 \mathrm{U}$ ou $2,0 \mathrm{~mL}$ de TB-A, totalizando 500U.

Em virtude da maior parte das pesquisas urológicas serem realizadas utilizando a medicação Botox $^{\circledR}$, na dose de 100 ou $200 \mathrm{U}$, e neste experimento estarmos usando a medicação Dysport ${ }^{\circledR}$, realizouse a equivalência dos produtos empregando a taxa de conversão de 1 unidade de Botox ${ }^{\circledR}$ para cada 2,5 unidades de Dysport ${ }^{\circledR}$ (ROSALES et al., 2006), promovendo, dessa maneira, o uso das mesmas doses preconizadas pelos trabalhos científicos avaliados. Entretanto, doses de 250 unidades não foram utilizadas, uma vez que seus resultados não foram significativos em estudo prévio (MOSTACHIO et al., 2012). Antes e após 4, 8 e 16 semanas da realização da orquiectomia e da administração da TB-A, os cães foram submetidos ao exame ultrassonográfico da próstata. As imagens e os dados foram obtidos por um único operador. Por essas imagens, avaliou-se o contorno, ecogenicidade, homogeneidade e presença ou ausência de pontos de necrose no parênquima prostático. Ainda mensurou-se o comprimento craniocaudal $(\mathrm{C})$, a largura transversa (L), e a altura dorsoventral (A) da próstata. Para cada dimensão, três mensurações foram obtidas e a média dos três valores calculados. O volume prostático (VP) foi estimado através da fórmula: $\mathrm{VP}=(1 / 2,6 \times \mathrm{C} \times \mathrm{L} \times \mathrm{A})+$ 1,8 (KAMOLPATANA et al., 2000).

Para a determinação das concentrações séricas de DHT e testosterona (mensuradas antes e após 4,8 e 16 semanas), três amostras de sangue foram coletadas entre $8 \mathrm{~h} 30 \mathrm{~min}$ e $11 \mathrm{~h} 30 \mathrm{~min}$, com 20 minutos de intervalo, centrifugadas e o plasma sanguíneo acondicionado em frascos plásticos e mantidos a $-20^{\circ} \mathrm{C}$. Os níveis de DHT e testosterona foram determinados por radioimunoensaio em fase sólida, utilizando-se kits comerciais. Todas as 
amostras foram analisadas em duplicata em teste único, utilizando uma única curva de calibração, não tendo assim variação interensaio. O coeficiente de variação intraensaio foi de $15 \%$.

O sêmen foi coletado por manipulação digital e analisado antes e após 2, 4, 8, e 16 semanas do tratamento com TB-A. A primeira fração foi desprezada, sendo a segunda e a terceira frações coletadas juntas em tubos plásticos graduados de $15 \mathrm{~mL}$. A análise seminal foi realizada imediatamente após a coleta. A avaliação do sêmen consistiu da mensuração do $\mathrm{pH}$, volume, motilidade espermática (escala de 0 a 100\%), vigor (escore de 0 a 5 , onde 0 representa nenhum movimento e 5 movimento vigoroso), concentração e morfologia espermática (expressa em porcentagem e classificada em defeitos maiores e menores). Pelo menos 100 células espermáticas foram analisadas. A libido foi avaliada subjetivamente com base na facilidade da coleta de sêmen (escala de 0 a 4 ), onde 4 representa rápida ereção e presença de movimentos pélvicos e 0 representa ausência de ereção e ejaculação.

Todas as análises estatísticas foram obtidas utilizando o programa SAS System (SAS System, version 9.0, Cary, NC, USA). Os dados foram submetidos à análise de variância (ANOVA), seguida pelo teste $t$ de Student pareado, empregado para comparação de médias entre os diferentes tempos dentro do mesmo grupo. O teste de Tukey foi usado para comparação de médias, entre os grupos, obtidas durante cada tratamento. Diferenças estatísticas foram consideradas significativas quando $\mathrm{P}<0,05$.

\section{RESULTADOS}

Os principais sinais clínicos observados antes do início dos tratamentos foram: hematúria, sangramento espontâneo pelo pênis e dificuldade de defecação, correspondendo estes a 55, 23 e $15 \%$ das alterações, respectivamente. Segundo os dados obtidos, os sinais clínicos observados pelos proprietários cessaram em média após uma e duas semanas do procedimento de orquiectomia e da administração de TB-A, respectivamente. O método de administração da TB-A mostrou-se seguro e nenhuma complicação local ou efeitos sistêmicos foram observados após a administração da neurotoxina. Áreas de hipoecogenicidade, as quais poderiam estar associadas à necrose nos locais de aplicação da TB-A, não foram observadas durante os exames ultrassonográficos da próstata.

$\mathrm{O}$ volume prostático não foi significativamente diferente entre o GI e GII antes dos tratamentos. A média do volume prostático dos animais do GI antes da orquiectomia era de
$40,49 \pm 28,31 \mathrm{~cm}^{3}$ (Tabela 1). Após quatro semanas, a ultrassonografia revelou significativa redução desse parâmetro. Comparando os dados obtidos com os valores iniciais, a média do volume prostático apresentou redução de $64,4 \%$. Na oitava e décima sexta semana, todas as variáveis observadas sofreram novas reduções, sendo estas significativas quando confrontadas aos valores basais. Após 16 semanas da orquiectomia, obteve-se uma redução de $80,5 \%$ do volume prostático dos animais do GI.

A média do volume prostático dos animais do GII no início do experimento era de $32,18 \pm 8,61 \mathrm{~cm}^{3}$ (Tabela 1). Após quatro semanas, o volume da próstata regrediu em todos os cães pertencentes a este grupo, causando uma redução significativa de 24,3\%. Na oitava semana de avaliação, o volume prostático, assim como os demais parâmetros analisados, permaneceram significativamente reduzidos, atingindo o volume a sua máxima redução $(29,8 \%)$ durante o período experimental. Após 16 semanas da administração da TB-A nos animais do GII, os resultados foram mantidos, permanecendo as reduções apresentadas pelo volume, comprimento e altura estatisticamente significativas.

A concentração sérica média de testosterona e DHT antes do tratamento nos animais do GI eram de $2,90 \pm 0,73 \mathrm{ng} \mathrm{mL}^{-1}$ e $0,71 \pm 0,34 \mathrm{ng}$ $\mathrm{mL}^{-1}$, respectivamente. Após quatro semanas da orquiectomia, os níveis de testosterona e DHT apresentaram significativa redução, quando comparado aos níveis pré-tratamento (de 2,90 0 , 73 para menos do que $0,1 \mathrm{ng} \mathrm{mL}^{-1}$ e de $0,71 \pm 0,34$ para $0,28 \pm 0,83 \mathrm{ng} \mathrm{mL}^{-1}$, respectivamente). Tais efeitos foram mantidos durante o período experimental. Os concentrações séricas de DHT e testosterona prétratamento nos animais do GII foram de $0,34 \pm 0,18 \mathrm{ng}$ $\mathrm{mL}^{-1}$ e $2,64 \pm 0,44 \mathrm{ng} \mathrm{mL}^{-1}$, respectivamente. Nenhuma diferença hormonal estatisticamente significativa foi observada após a aplicação de TB-A.

Todos os animais pertencentes ao GII não apresentaram alterações na libido, ereção e ejaculação durante as coletas de sêmen. Nenhuma diferença significativa foi observada nos parâmetros seminais, incluindo volume, motilidade, vigor, concentração, $\mathrm{pH}$, e morfologia após a administração da TB-A, durante as 16 semanas de avaliação (Tabela 2).

\section{DISCUSSÃO}

Em vista do grande número de hipóteses sobre a patogênese desta condição, o tratamento da HPB está constantemente em transição. Embora a orquiectomia continue sendo o tratamento de escolha, esta nem sempre pode ser indicada a pacientes de alto risco ou destinados à reprodução (MOSTACHIO et al., 2012). Nesse contexto, 
Tabela 1 - Média dos valores ( \pm desvio padrão) dos parâmetros prostáticos no início e após 4,8 e 16 semanas da orquiectomia (GI) ou administração de $500 \mathrm{U}$ de toxina botulínica A (GII).

\begin{tabular}{|c|c|c|c|c|c|}
\hline Grupo & Semanas & Comprimento $(\mathrm{cm})$ & Altura $(\mathrm{cm})$ & Largura $(\mathrm{cm})$ & Volume $\left(\mathrm{cm}^{3}\right)$ \\
\hline \multirow{4}{*}{ I } & 0 & $4,73 \pm 1,57^{\mathrm{A}}$ & $3,64 \pm 1,27^{\mathrm{A}}$ & $4,89 \pm 1,21^{\mathrm{A}}$ & $40,49 \pm 28,3^{\mathrm{A}}$ \\
\hline & 4 & $2,90 \pm 1,30^{\mathrm{B}}$ & $2,61 \pm 1,00^{\mathrm{AB}}$ & $3,08 \pm 0,95^{\mathrm{B}}$ & $13,60 \pm 9,95^{\text {В }}$ \\
\hline & 8 & $2,42 \pm 0,99^{\mathrm{B}}$ & $2,17 \pm 0,80^{\mathrm{B}}$ & $2,66 \pm 0,77^{\mathrm{B}}$ & $8,58 \pm 5,53^{\text {В }}$ \\
\hline & 16 & $2,28 \pm 0,93^{\mathrm{B}}$ & $2,13 \pm 0,78^{\mathrm{B}}$ & $2,53 \pm 0,72^{\mathrm{B}}$ & $7,88 \pm 5,03^{\text {в }}$ \\
\hline \multirow{4}{*}{ II } & 0 & $3.94 \pm 0.44^{\mathrm{A}}$ & $4.02 \pm 0.39^{\mathrm{A}}$ & $4.90 \pm 0.44^{\mathrm{A}}$ & $32.18 \pm 8.61^{\mathrm{A}}$ \\
\hline & 4 & $3.56 \pm 0.35^{\mathrm{AB}}$ & $3.54 \pm 0.35^{\mathrm{B}}$ & $4.59 \pm 0.34^{\mathrm{A}}$ & $24.36 \pm 5.76^{\mathrm{B}}$ \\
\hline & 8 & $3.49 \pm 0.33^{\mathrm{B}}$ & $3.43 \pm 0.33^{\mathrm{B}}$ & $4.44 \pm 0.41^{\mathrm{A}}$ & $22.58 \pm 5.53^{B}$ \\
\hline & 16 & $3.51 \pm 0.29^{\mathrm{B}}$ & $3.43 \pm 0.33^{\mathrm{B}}$ & $4.46 \pm 0.44^{\mathrm{A}}$ & $22.74 \pm 5.53^{B}$ \\
\hline
\end{tabular}

Médias na mesma coluna com uma letra maiúscula em comum não diferem significamente entre si $(\mathrm{P}<0,05)$.

a experimentação de novos fármacos, como a TB-A, está assumindo importante papel nesse cenário.

A injeção de TB-A não provocou o surgimento de qualquer sinal de botulismo nos cães estudados, apesar da extensa irrigação sanguínea presente na próstata, a qual, em teoria, poderia facilitar a difusão sistêmica da neurotoxina. Tal fato e descrição corroboram os encontrados por MARIA et al. (2003) e CHUANG et al. (2006a), que sugerem que a TB-A é extremamente segura quando utilizada para o tratamento da HPB.

Neste estudo, a queixa principal dos proprietários, assim como a sintomatologia clínica observada durante o experimento, foi hematúria, seguida por gotejamento de fluído uretral não associado à urina e dificuldade de defecação. Os dados apresentados corroboram os descritos por READ \& BRYDEN (1995), que descreveram que a maior parte dos animais com HPB apresentam hematúria e ou sangramento espontâneo pelo pênis como os principais motivos do atendimento médico, sendo que tais alterações podem ser devidas à presença de processo inflamatório e, consequentemente, ao aumento da vascularização prostática que a liberação de linfócitos e interleucinas promovem.

BARSANTI \& FINCO (1995) descreveram que, após sete dias da orquiectomia em animais com HPB, iniciou-se a involução da próstata e diminuição dos sinais clínicos. Depois de três e nove semanas, o volume do órgão havia regredido 50 e 75\%, respectivamente. Nossos dados estão de acordo com os descritos, pois os sinais clínicos cessaram depois de uma semana da orquiectomia. Após 16 semanas desta, obtivemos uma redução de $80,5 \%$ do volume prostático. Como a próstata é um órgão andrógenodependente e ambos os compartimentos (estromal e glandular) são sensíveis a este, a resolução e decréscimo das variáveis ocorreram em função da

Tabela 2 - Valores médios ( \pm desvio padrão) dos parâmetros espermáticos dos ejaculados de 8 cães submetidos à administração de 500 U de toxina botulínica A.

\begin{tabular}{|c|c|c|c|c|c|}
\hline \multirow{2}{*}{ Parâmetros Seminais } & \multirow[b]{2}{*}{0} & \multirow[b]{2}{*}{2} & \multirow[b]{2}{*}{4} & \multirow[b]{2}{*}{8} & \multirow[b]{2}{*}{16} \\
\hline & & & & & \\
\hline Volume (mL) & $4,9 \pm 1,7$ & $4,2 \pm 0,7$ & $4,5 \pm 0,9$ & $4,3 \pm 1,1$ & $4,2 \pm 1,0$ \\
\hline Motilidade (\%) & $87.7 \pm 4.7$ & $86,2 \pm 3,9$ & $88.7 \pm 3.5$ & $88.2 \pm 3.9$ & $89.0 \pm 3.1$ \\
\hline Vigor & $4.0 \pm 0.1$ & $3,9 \pm 0,1$ & $4.0 \pm 0.3$ & $4.0 \pm 0.3$ & $4.1 \pm 0.3$ \\
\hline Concentração $\left(\times 10^{6} \mathrm{~mL}^{-1}\right)$ & $190.0 \pm 86.7$ & $194,7 \pm 76,4$ & $178.6 \pm 56.3$ & $181.2 \pm 48.0$ & $169.2 \pm 52.3$ \\
\hline $\mathrm{pH}$ & $6.4 \pm 0.2$ & $6,3 \pm 0,2$ & $6.3 \pm 0.2$ & $6.3 \pm 0.1$ & $6.3 \pm 0.1$ \\
\hline Sptz normais $(\%)$ & $70.8 \pm 11.6$ & $68,2 \pm 11,3$ & $70.2 \pm 13.0$ & $70.8 \pm 10.8$ & $72.0 \pm 12.2$ \\
\hline Sptz com defeitos maiores (\%) & $11.5 \pm 8.1$ & $13,0 \pm 9,6$ & $11.3 \pm 9.8$ & $11.3 \pm 9.2$ & $10.7 \pm 7.3$ \\
\hline Sptz com defeitos menores $(\%)$ & $17,7 \pm 5.4$ & $18,8 \pm 4,2$ & $18.5 \pm 4.5$ & $17.8 \pm 4.1$ & $17.3 \pm 5.9$ \\
\hline
\end{tabular}

Valores não diferem significamente entre si $(\mathrm{P}<0,05)$.

Sptz: Espermatozoides.

Ciência Rural, v.45, n.9, set, 2015. 
queda que a orquiectomia promoveu nos níveis hormonais. Por outro lado, a administração de TB-A não acarretou alterações significativas nos níveis de testosterona e DHT nos animais pertencentes ao GII, possuindo, portanto uma ação indireta sobre a próstata. No estudo realizado por KUO (2003), observou-se melhora dos sinais após a aplicação de TB-A entre o sétimo e décimo quarto dia. Em nosso estudo, dados semelhantes foram obtidos, ocorrendo melhora em média após 10 dias do início do tratamento.

Apesar do pequeno número de animais estudados, a redução do volume da glândula confirma as observações prévias descritas por outros pesquisadores (DOGGWEILER et al., 1998; KUO, 2003). O decréscimo observado neste experimento não foi o mesmo atingido por MARIA et al. (2003) ou CHUANG et al. (2006a), os quais conseguiram uma diminuição superior a $50 \%$ em homens e ratos, respectivamente. Entretanto, os dados aqui apresentados para o GII estão de acordo com os encontrados por LIN et al. (2007), que relataram que, após um mês da aplicação de $200 \mathrm{U}$ de TB-A na próstata de cães, ocorreu uma diminuição de $24 \%$ no tamanho do órgão. No presente estudo, a avaliação da resposta à aplicação de TB-A após quatro semanas mostrou decréscimo muito semelhante $(24,3 \%)$. Por outro lado, notamos uma maior depleção após oito e 16 semanas, atingindo valores de 29,8 e $29,3 \%$, respectivamente. Uma explicação para essa variedade seria a utilização de diferentes doses de toxina botulínica e a desigualdade entre as proporções dos compartimentos prostáticos (glandular e estromal) nas diferentes espécies (CHUANG et al., 2006b). A HPB humana é nodular e envolve vários tipos celulares, sendo que esta é caracterizada primariamente por proliferação dos elementos estromais da glândula. Por outro lado, nos cães, o crescimento prostático envolve os dois compartimentos (JOHNSTON et al. 2001). Outra possível elucidação seria o fato de que diferentes indivíduos da mesma espécie apresentam disparidades individuais quanto à capacidade de regeneração neurológica (CHUANG et al., 2006b).

A observação de pontos de necrose nos locais de aplicação da TB-A pela ultrassonografia poderia explicar a redução do volume, entretanto, as avaliações ultrassonográficas não evidenciaram tal alteração. Essa visualização confirma os dados relatados no que concerne à ausência de necrose nas áreas de injeção da TB-A (KUO 2005). Segundo alguns autores, a razão pela qual a terapia com TB-A promove decréscimo do tamanho da próstata seria a ocorrência de morte celular programada, a qual estaria relacionada a uma ação indireta da toxina (denervação) sobre a próstata(CHUANGetal.,2006b).

Segundo PENNEFATHER et al. (2000), a atividade neuronal está envolvida no crescimento prostático, assimcomoemsuas funções. Adesregulação dessa atividade, poderia alterar a morfologia e o comportamento da próstata, podendo resultar na HPB. Portanto, a normalização dessa alteração neuronal, com a inibição da liberação de acetilcolina, seria capaz de reverter significativamente o processo. Em adição, pode ocorrer uma depleção na produção de fatores de crescimento epidermal, os quais possuem atividades tróficas, em virtude desta denervação. Dessa maneira, é admissível que parte dos resultados com a TB-A seja consequência da intoxicação das fibras simpáticas e queda da atividade adrenérgica.

Os resultados obtidos no presente estudo estão em concordância com os relatados por MARIA et al. (2003) quanto à ocorrência de máxima depleção do volume prostático, após dois ou três meses da injeção de TB-A. Da mesma maneira, os dados aqui descritos estão de acordo com os mesmos autores, no que se refere ao período de efetividade da TB-A, onde estes apresentam declínio após quatro a seis meses. Todavia, é incerto por quanto tempo a redução do volume prostático permanecerá em níveis aceitáveis, assim como a ausência de sinais clínicos, sendo necessários novos estudos.

No que diz respeito à comparação entre os tratamentos, a orquiectomia evidenciou resultados muito superiores aos dos cães tratados com TB-A. Tal discrepância está relacionada ao fato de que, com a retirada dos testículos, as concentrações hormonais atingem níveis mínimos na circulação, levando a uma redução da próstata. Por outro lado, CHUANG et al. (2006a) descreve que a TB-A não afeta a expressão dos receptores de andrógenos no tecido prostático, permanecendo intacta a capacidade de resposta à influência trófica da testosterona e DHT. Essa habilidade poderia, portanto, causar uma menor diminuição das variáveis prostáticas e também explicar em parte a menor duração dos efeitos sobre o órgão.

No presente estudo, nenhum efeito sobre a libido e ereção foram observados, uma vez que os níveis hormonais permaneceram normais após o tratamento com TB-A. Os dados deste estudo estão de acordo com os descritos por LIN \& QUEK (2008) e previamente por nosso grupo (MOSTACHIO et al., 2012), os quais não mencionaram nenhuma disfunção ejaculatória ou de fertilidade, respectivamente, após a administração de TB-A. Neste estudo, não foram observadas alterações nas características e morfologia espermática após a administração de TB-A. No 
entanto, os dados do presente estudo mostraram ainda que a morfologia espermática encontra-se abaixo dos valores de normalidade para a espécie. Isso pode estar relacionado à idade dos animais, uma vez que os cães com idade superior a sete anos apresentaram maior número de defeitos espermáticos, quando comparados aos mais novos. Aspectos nutricionais também podem estar envolvidos, pois a alimentação fornecida pelos proprietários era de baixa qualidade. Entretanto, futuros estudos avaliando as implicações in vitro e in vivo da administração de TB-A são de fundamental importância.

\section{CONCLUSÃO}

O presente trabalho confirma a capacidade da TB-A em reduzir o volume prostático e sugere ser um tratamento alternativo eficaz e sem efeitos colaterais em animais destinados ou não à reprodução com HPB. No entanto, tais efeitos são inferiores e de menor duração quando comparados aos alcançados após a orquiectomia.

\section{AGRADECIMENTOS}

À Fundação de Amparo à Pesquisa do Estado de São Paulo (FAPESP), pela bolsa (2005/58339-2) e auxílio à pesquisa (2006/52616-1) concedidos.

\section{COMITÊ DE ÉTICA}

Protocolo número 012292-06.

\section{REFERÊNCIAS}

BARSANTI, J.A.; FINCO, D.R. Medical management of canine prostatic hyperplasia. In: BONAGURA, J.D.; KIRK, R.W. Current veterinary therapy XII. Philadelphia: Saunders, 1995. p.1033-1034.

CHUANG, Y.C. et al. Novel action of botulinum toxin on the stromal and epithelial components of the prostate gland. Journal of Urology, v.175, p.1158-1163, 2006a. Disponível em: <http:// www.jurology.com/article/S0022-5347(05)00318-6/abstract $>$. Acesso em: 22 maio 2014. doi: 10.1016/S0022-5347(05)00318-6.

CHUANG Y.C. et al. Intraprostatic injection of botulinum toxin type-A relieves bladder outlet obstruction in human and induces prostate apoptosis in dogs. BMC Urology, v.6, p.12, 2006b. Disponível em: <http://www.biomedcentral.com/content/ pdf/1471-2490-6-12.pdf $>$. Acesso em: 22 maio 2014. doi: $10.1186 / 1471-2490-6-12$

DOGGWEILER, R. et al. Botox-induced prostatic involution. Prostate, v.37, p.44-55, 1998. Disponível em: $<$ http://onlinelibrary.wiley.com/doi/10.1002/(SICI)10970045(19980915)37:1\%3C44::AID-PROS7\%3E3.0.CO;2-8/ abstract>. Acesso em: 22 maio 2014. doi: 10.1002/(SICI)10970045(19980915)37:1<44::AID-PROS7>3.0.CO;2-8.
JOHNSTON, S.D. et al. Disorders of the canine prostate. In: Canine and feline theriogenology. Philadelphia: Saunders, 2001. p.337-355.

KAMOLPATANA K. et al. Determination of canine prostatic volume using transabdominal ultrasonography. Veterinary Radiology \& Ultrasound, v.41, p.73-77, 2000. Disponível em: $<$ http://onlinelibrary.wiley.com/doi/10.1111/j.1740-8261.2000. tb00430.x/abstract>. Acesso em: 22 maio 2014. doi: 10.1111/ j.1740-8261.2000.tb00430.x.

KUO, H.C. Effect of botulinum A toxin in the treatment of voiding dysfunction due to detrusor underactivity. Urology, v.61, p.550554, 2003. Disponível em: <http://www.goldjournal.net/article/ S0090-4295(02)02541-4/abstract>. Acesso em: 22 maio 2014. doi: 10.1016/S0090-4295(02)02541-4.

KUO, H.C. Prostate botulinum A toxin injection - an alternative treatment for benign prostatic obstruction in poor surgical candidates. Urology, v.65, p.670-674, 2005. Disponível em:<http:// www.goldjournal.net/article/S0090-4295(04)01334-2/abstract>. Acesso em: 22 maio 2014. doi: 10.1016/j.urology.2004.10.077.

LIN, A.T. et al. Effects of botulinum toxin A on the contractile function of dog prostate. European Urology, v.52, p.582-589, 2007. Disponível em: <http://www.sciencedirect.com/science/ article/pii/S0302283807003557>. Acesso em: 22 maio 2014. doi: 10.1016/j.eururo.2007.03.002.

LIN, S.K.; QUEK, P.L. Intraprostatic and bladder-neck injection of botulinum A toxin in treatment of males with bladder-neck dyssynergia: a pilot study. European Urology, v.53, p.620-627, 2008. Disponível em: <http://www.sciencedirect.com/science/ article/pii/S0302283807012523>. Acesso em: 22 maio 2014. doi: 10.1016/j.eururo.2007.10.001.

MARIA, G. et al. Relief by botulinum toxin of voiding dysfunction due to benign prostatic hyperplasia: results of a randomized, placebo-controlled study. Urology, v.62, p.259-265, 2003. Disponível em: $\quad<$ http://www.goldjournal.net/article/S00904295(03)00477-1/abstract>. Acesso em: 22 maio 2014. doi: 10.1016/S0090-4295(03)00477-1.

MOSTACHIO, G.Q. et al. Intra-prostatic injection of botulinum toxin type $A$ in treatment of dogs with spontaneous benign prostatic hyperplasia. Animal Reproduction Science, v.133, p.224-228, 2012. Disponível em: <http://www.animalreproductionscience. com/article/S0378-4320(12)00203-5/abstract>. Acesso em: 22 maio 2014. doi: 10.1016/j.anireprosci.2012.06.024.

PENNEFATHER, J.N. et al. The autonomic and sensory innervation of the smooth muscle of the prostate gland: a review of pharmacological and histological studies. Journal of Autonomic Pharmacology, v.20, p.193-206, 2000. Disponível em: <http:// onlinelibrary.wiley.com/doi/10.1046/j.1365-2680.2000.00195.x/ abstract>. Acesso em: 22 maio 2014. doi: 10.1046/j.13652680.2000.00195.x

READ, R.A.; BRYDEN, S. Urethral bleeding as a presenting sign of benign prostatic hyperplasia in the dog: a retrospective study (1979-1993). Journal of the American Animal Hospital Association, v.31, p.261-267, 1995. Disponível em: <http://www. jaaha.org/doi/abs/10.5326/15473317-31-3-261>. Acesso em: 23 out. 2014. doi: 10.5326/15473317-31-3-261.

ROSALES, R.L. etal. Pharmacology of botulinum toxin: differences between type A preparations. European Journal of Neurology, v.13, p.2-10, 2006. Disponível em: < http://onlinelibrary.wiley. com/doi/10.1111/j.1468-1331.2006.01438.x/abstract $>$. Acesso em: 23 out. 2014. doi: 10.1111/j.1468-1331.2006.01438.x. 